\title{
Serial Search Based Code Acquisition in the Cooperative MIMO Aided DS-CDMA Downlink
}

\author{
SeungHwan Won, Kyungchun Lee and Lajos Hanzo \\ School of ECS, Univ. of Southampton, SO17 1BJ, UK. \\ Tel: +44-23-8059 3125, Fax: +44-23-8059 4508 \\ E-mail: $\{$ shw04r, kl7v, lh\} @ecs.soton.ac.uk \\ http://www-mobile.ecs.soton.ac.uk
}

\begin{abstract}
In this paper we investigate a realistic code acquisition assisted cooperative Non-Coherent (NC) Multiple-Input Multiple-Output (MIMO) DS-CDMA downlink scenario, when communicating over uncorrelated single-path and multi-path Rayleigh channels. The probabilities of correct detection and false alarm have been derived analytically. Furthermore, a Mean Acquisition Time (MAT) formula is provided for the cooperative transmission scenario considered. The associated MAT performance trends are characterised as a function of both the number of Relay Stations (RSs), as well as that of the receive antennas and link imbalance. As opposed to the classic scenario of having co-located MIMO elements, our findings suggest that employing distributed MIMO elements acting as RSs combined with multiple receive antennas leads to an improved MAT performance.
\end{abstract}

\section{INTRODUCTION}

In wireless networks, fading constitutes one of the main sources of channel-induced impairments. A powerful technique of overcoming the fading imposed by multi-path propagation is constituted by spatial diversity invoking multiple transmit/multiple receive antennas, which has attracted substantial research interests [1]. However, in realistic propagation environments, the multiple antenna's signals typically become correlated owing to the size-limitation of the Mobile Station (MS) and Base Station (BS). Hence the spatial diversity gain of independently faded signals is often eroded. This phenomenon is typically imposed by shadow fading. In order to cope with this problem, various cooperative and RS-aided transmission schemes have been proposed [2], [3], [4]. In low-complexity cooperative systems a MS receives the two-hop DownLink (DL) signal via the RS as well as the directly detected DL signal of the BS. Since these two signals generally arrive through completely different -rather than correlated- propagation paths, cooperative transmission becomes capable of mitigating the above-mentioned correlated shadow fading effects. Furthermore, exploiting the intermediate RS, cooperative transmission has the potential of extending the cell area and/or of improving the quality of cell-edge coverage, which results in requiring a reduced number of BSs. In cooperative or distributed MIMO scenarios, the RS may be constituted by an intermediate MS that is currently not engaged in active communication or by a fixed RS that is installed at a specific position in a cell. However, for the sake of creating high-reliability relay systems, in this treatise we consider fixed RSs. It is also assumed that there is a Line-Of-Sight (LOS) path between the RS as well as the BS [4] and that the required initial procedures have been completed, before commencing the first stage of RS-aided code acquisition.

The financial support of the Ministry of Information and Communication (MIC), Republic of Korea, the Royal Society,UK and of the European Union as well as that of the EPSRC is gratefully acknowledged.
In inter-cell synchronous CDMA systems the MS's receiver must be capable of synchronously aligning a locally generated PseudoNoise (PN) code with the received composite multi-user signal containing the desired user's PN sequence within an allowable timing error. Substantial research efforts have also been devoted to the design of code acquisition techniques [5]. These efforts have been extended to code acquisition schemes designed for MIMO systems having co-located antenna elements [6], [7]. These results suggest that increasing the number of transmit antennas in a NC MIMO-aided CDMA system having co-located antenna elements typically results in a MAT performance degradation, due to sharing the total transmit power across several MIMO elements regardless whether single-path or multi-path scenarios are considered [6], [7]. Since there are no in-depth studies representing the fundamental characteristics of code acquisition schemes in RS-aided scenarios in the context of multi-path propagation scenarios, solving this open problem is the objective of the present contribution. Against this background, in this treatise we investigate serial search based code acquisition schemes designed for fixed RS-aided scenarios. More explicitly, we quantify both the attainable correct detection probability $\left(P_{D}\right)$ as well as the false alarm probability $\left(P_{F}\right)$ performance as a function of both the Signal-to-Interference plus Noise Ratio (SINR) per chip $\left(E_{c} / I_{0}\right)$ and that of the number of receive antennas $R$. Furthermore, we characterise the attainable MAT versus $E_{c} / I_{0}$ performance parameterised by the grade of link imbalance as well as by the number of receive antennas and RSs in both single- and multi-path scenarios. This paper is organised as follows. Section II describes the system investigated, followed by the correct detection and false alarm probability analysis of code acquisition schemes in the context of uncorrelated Rayleigh channels, whilst the MAT analysis of fixed RS-aided scenarios is illustrated in Section III. In Section IV, our numerical MAT results are discussed, while our conclusions are offered in Section V.

\section{CORRECT DETECTION AND FALSE ALARM PROBABILITY}

Fig.1 illustrates a number of code acquisition scenarios encountered in fixed RS-aided environments, when considering one or two RSs. The traffic cell is divided into three sectors and a fixed RS is employed in each sector. We assume that both the BS and the RS have a single transmit antenna. As portrayed in the bottom centre sector of Fig.1, the MS receives the DL signals from both the RS located at the same sector's centre as well as from the BS and processes them for the sake of attaining reliable code acquisition. On the other hand, when the MS is located at the edge of the top left sector of Fig.1, the signal received from the RS at the adjacent sector's centre seen at the bottom of Fig.1 also arrives at the MS with a substantial 


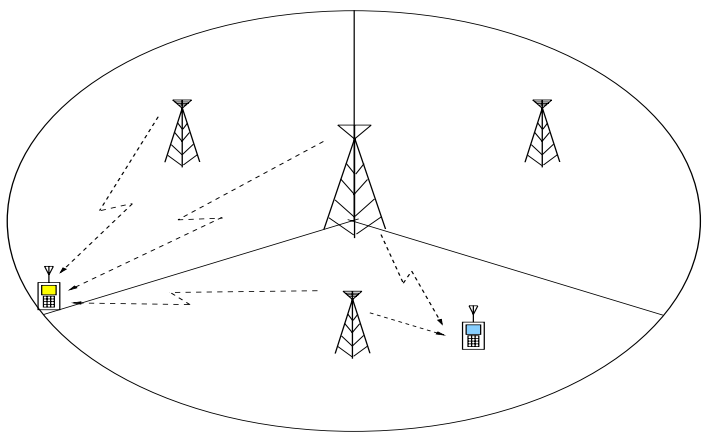

Fig. 1. Code acquisition scenarios in cooperative MIMO environments, when considering one or two RSs

signal strength. Therefore, in this case, this particular MS has to combine the signals received from all the three transmitters for the sake of reliable code acquisition. The average strength of the RS' signal varies depending on the location of the MS. Explicitly, when the MS is located near the cell-edge, the signal received from the RS generally has a higher signal strength with respect to that of the BS, which results in the phenomenon often referred to as link imbalance between the signals received by the MS.

We assume that a finite-length tapped delay line channel model generates $L$ independently Rayleigh-faded multi-path signals, each arriving with a time delay $\tau_{l}$ having a tap spacing of one chipduration [8], where $l=L$ is the number of multi-path components encountered. It is also assumed that the Rayleigh fading is sufficiently slow for the faded envelope to remain constant over the duration of $\tau_{D}=N \cdot T_{c}$, but sufficiently rapid so that the consecutive $\tau_{D}$-duration segments may be considered essentially independently faded, as in [9], where $\tau_{D}$ indicates the integral dwell time, $N$ represents the number of chips accumulated over the duration of $\tau_{D}$ and $T_{c}$ is the chip duration. The fixed RS-aided DS-CDMA DL signal received over the multi-path Rayleigh fading channel considered may be expressed as [6]

$$
\begin{array}{r}
r(t)=\sum_{p=1}^{P} \sum_{l=1}^{L} \sum_{r=1}^{R}\left[\alpha_{(p, l, r)} \sqrt{\frac{E_{c}}{T_{c}}} c\left(t+d T_{c}+\tau_{p}+\tau_{l}\right)\right. \\
\left.\cdot w_{p}\left(t+d T_{c}+\tau_{p}+\tau_{l}\right) \cdot \exp \left(2 \pi f t+\phi_{(p, l, r)}\right)+I_{(p, l, r)}(t)\right],
\end{array}
$$

where $p=P$ is the number of RSs, $r=R$ is the number of receive antennas, and $\alpha_{(p, l, r)}$ represents the complex-valued envelope of the $(p, l, r)^{t h}$ signal path obeying the Rayleigh distribution. Furthermore, $E_{c}$ denotes the pilot signal energy per PN code chip, $c(t)$ represents a common PN sequence having a cell-specific code-phase offset, while $d$ is the code phase offset with respect to the phase of the local code. Still considering Eq. $1, w_{p}(t)$ identifies the specific Walsh code assigned to the $p^{t h} \mathrm{RS}, \tau_{p}$ indicates the relative time difference of the signal received from a RS with respect to the signal received from the BS, $f$ is the carrier frequency and finally, $\phi$ is the carrier phase of a specific user's modulator. Furthermore, $I_{(p, l, r)}(t)$ is the complex-valued AWGN having a double-sided power spectral density of $I_{0}$ at the $(p, l, r)^{t h}$ path. For the sake of a fair comparison, it is assumed that in the absence of link imbalance, the total power received by the MS in RS-aided scenarios is the same as that of the corresponding multiple receive antenna assisted scenarios. Fig.2 depicts the block diagram of the NC DS-CDMA DL receiver designed for our code acquisition scheme using $R$ co-located receive antennas. The receiver generates a decision variable by accumulating $R$ number of independently faded signals observed over a time interval for the sake of improving the $P_{D}$ in the mobile channel imposing both fading and poor SINR conditions. By employing the procedures proposed in [6] in the context of the receiver structure of Fig.2, the final decision variable may be written as

$$
Z_{(k, p)}=\sum_{r=1}^{R}\left\|\frac{1}{\sqrt{2}} \cdot\left(\sqrt{\frac{4 E_{c}}{N I_{0}}} \cdot S_{(k, p)(r)}+I_{(k, p)(r)}\right)\right\|^{2},
$$

where $k$ denotes the $k^{t h}$ chip's sampling instant, $S_{(k, p)(r)}$ is assumed to be a deterministic value, which depends on whether a signal is present or absent as described in [6], $\|\cdot\|^{2}$ represents the Euclidean norm of the complex-valued argument and the factor of $1 / \sqrt{2}$ is employed to appropriately normalise the noise variance. Accordingly, the decision variable $Z_{(k, p)}$ obeys a noncentral chi-square Probability Density Function (PDF) with $2 R$ degrees of freedom, where the noncentrality parameter $\lambda_{x}$ is either $2 N\left(\frac{E_{c}}{I_{0}}\right)$ for the hypothesis of the desired signal being present $\left(H_{x}, x=1\right)$ or $\frac{2}{N}\left(\frac{E_{c}}{I_{0}}\right)$ for it being absent $\left(H_{x}, x=0\right)$ [6]. The effects of both timing errors and frequency mismatches are encapsulated by the definition of $\left(E_{c} / I_{0}\right)^{\prime}$ [7] and the corresponding noncentrality parameter, $\overline{\lambda_{x}} \equiv \lambda_{x} \sigma^{2}$ is either $2 N\left(\frac{\overline{E_{c}}}{I_{0}}\right)^{\prime}$ when the desired signal is deemed to be present $(x=1)$ or $\frac{2}{N}\left(\frac{\overline{E_{c}}}{I_{0}}\right)^{\prime}$ when it is deemed to be absent $(x=0)$. We also define $\mu_{x}=\left(2+\overline{\lambda_{x}}\right)$, which physically represents a new biased noncentrality parameter. Further details on the related calculations and derivations can be found in [6], [7]. When using
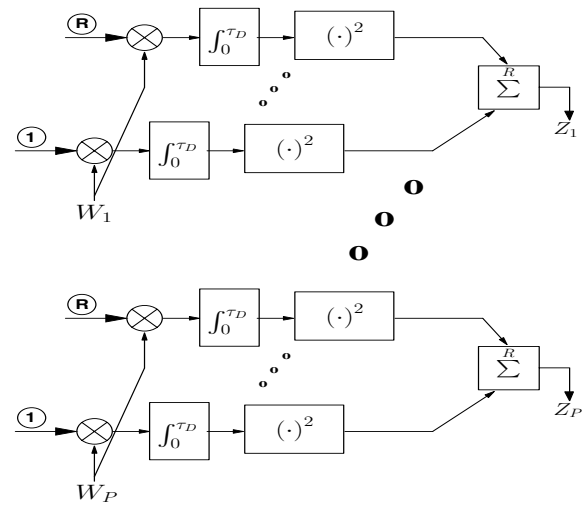

Fig. 2. Receiver structure of a NC code acquisition system employing $R$ receive antennas in RS-aided scenarios

the procedures outlined in [6], finally, the decision variables $Z_{(k, p)}$ are constituted by the sum of $R$ number of independent variables according to $Z_{(k, p)}=\sum_{r=1}^{R} Z_{(k, p)(r)}$, whilst the desired signal's PDF may be formulated using the procedure outlined in [6], [7] as:

$$
f_{Z_{(k, p)}}\left(z \mid H_{x}\right)=\frac{z^{(R-1)} e^{-z / \mu_{x}}}{\Gamma(R) \cdot \mu_{x}^{R}},
$$

where $\Gamma(\cdot)$ is the Gamma function. Then, the $P_{D}$ or $P_{F}$ corresponding to $x=1$ or 0 , respectively, may be expressed as

$$
\left.P\right|_{\xi=D \text { or } F}=\int_{\theta}^{\infty} f_{Z_{(k, p)}}\left(z \mid H_{x}\right) d z
$$




$$
=\exp \left(-\frac{\theta}{\mu_{x}}\right) \cdot \sum_{r=0}^{R-1} \frac{\left(\theta / \mu_{x}\right)^{r}}{r !}
$$

where $\theta$ is a threshold value. By employing Eq.5, the transfer functions required for calculating the achievable MAT of the fixed RS-aided scenarios considered will be derived in the forthcoming section.

\section{MAT ANALYSIS OF CODE ACQUISITION IN RS-AIDED SCENARIOS}

The classic serial search techniques designed for code acquisition [6], [7] have been traditionally employed in specific scenarios, where the uncertainty region (or search window width) is quite wide, namely on the order of the code length, such as $\left(2^{15}-1\right)$ chips. The length of the PN sequence in our system was also assumed to be $\left(2^{15}-1\right) \cdot T_{c}$, as in the DL of the inter-cell synchronous CDMA-2000 system [6], [7] for example, where the chip-duration is $T_{c}=1 / 1.2288 \mu \mathrm{s}$. In the case of code acquisition contrived for DS-CDMA DL, the main design goal is to acquire coarse timing of the first received signal path impinging at the receiver, since this timing information is used as that of the reference finger of the Rake receiver. In [9], [10], explicit MAT formulae were provided for a single-antenna aided serial search based code acquisition scheme. There is a difference between a single- and co-located MIMO-aided schemes as well as a RS-assisted one in terms of analysing the MAT, because a signal recevied from a $\mathrm{RS}$ is a time delayed version of that directly received from the BS. Hence the independently fading multiple signal replicas received from RSs cannot be directly combined, as opposed to the colocated MIMO-element scenarios. Groups of multi-path components received from a BS and $\mathrm{RS}$ (s) may or may not overlap, depending on the path delays experienced. However, for the sake of simplifying our analysis, we assume that every group of multi-path components is non-overlapping and each arrives with a time delay that is an integer multiple of the chip-duration $\tau_{p}$, because the derivation of exact MAT formula can be simplified without grave inaccuracies, when the uncertainty region may be assumed to be quite wide, such as $\left(2^{15}-1\right)$ chips ${ }^{1}$. In terms of deriving both the $P_{D}$ and the $P_{F}$ based upon invoking multiple receive antennas, we will commence our discourse by analysing the MAT performance of code acquisition schemes employing Double Dwell Serial Search (DDSS) [10]. We assume that in each chip duration $T_{c}, \alpha$ number of correct timing hypotheses are tested instead of just a single one, which are spaced by $T_{c} / \alpha$. Hence the uncertainty region's total time-duration remains the same, but the number of legitimate locking positions to be tested is increased by a factor of $\alpha$. Moreover, as mentioned in Section II, when the $L$ multi-path signals arrive at time delays, which are integer multiples of the chip-duration $\tau_{l}$, then the relative frequency of the signal being present is increased $L$-fold. Similarly, the relative frequency of the signal being present is increased by a factor of $P$, when relying on $P$ RSs. The required transfer functions [9], [10], are defined as follows. The entire successful detection related transfer function $H_{D}(z)$ encompasses all the branches of a state diagram

\footnotetext{
${ }^{1}$ To elaborate a little further, when the uncertainty region is $\left(2^{15}-1\right)$ chip durations, the ratio between the number of chips in the uncertainty region and that of the legitimate locking positions corresponding to the signal being absent and present, respectively, is at most on the order of $10^{-4}$, which allows us to employ our aforementioned assumptions in the derivation of the MAT formula.
}

[9], [10], which lead to successful detection. Furthermore, $H_{0}(z)$ indicates the absence of the desired user's signal at the output of the acquisition scheme, whilst $H_{M}(z)$ represents the overall miss probability of a search run carried out across the entire uncertainty region. The related processes are detailed for DDSS in [10]. Then, it may be shown that the generalised expression derived for computing the MAT of the DDSS scheme is given by [8], [9]:

$$
\begin{array}{r}
E\left[T_{A C Q}\right]=\frac{1}{H_{D}(1)}\left[H_{D}{ }^{\prime}(1)+H_{M}{ }^{\prime}(1)+\right. \\
\left.\left\{(\nu-2 \alpha L P)\left[1-\frac{H_{D}(1)}{2}\right]+\frac{1}{2} H_{D}(1)\right\} H_{0}{ }^{\prime}(1)\right] \cdot \tau_{D 1} \\
\approx \frac{\left(1+H_{M}(1)\right) \cdot H_{0}{ }^{\prime}(1)}{2 \cdot\left(1-H_{M}(1)\right)} \cdot\left(\nu \cdot \tau_{D 1}\right),
\end{array}
$$

where $\left.H_{x}^{\prime}(z)\right|_{x=D, M \text { or } 0}$ is a derivative of $\left.H_{x}(z)\right|_{x=D, M \text { or } 0, \nu}$ represents the total number of uncertainty positions to be searched and $\tau_{D 1}$ denotes the $1^{\text {st }}$ dwell time. Again, the exact MAT formula can be simplified, if $\nu$ is significantly higher than the number of $H_{1}$ states, where $H_{1}$ represents that the signal may be deemed to be present [8]. Therefore, in order to simplify our numerical performance analysis, we adopted the specific approximation of the exact MAT expression proposed in [8]. Explicitly, since each resolvable path contributes two $H_{1}$ hypotheses and because the average $P_{D}$ associated with these two hypotheses is the same, the overall miss probability of the DDSS scheme in the fixed RS-aided scenario may be expressed as:

$H_{M}(1)=\prod_{p=1}^{P} \prod_{l=1}^{L} \prod_{\zeta=1}^{\alpha}\left[\left(1-P_{D 1(p, l, \zeta)}\right)+P_{D 1(p, l, \zeta)} \cdot\left(1-P_{D 2(p, l, \zeta)}\right)\right]^{2}$,

where $\left.P_{D x(p, l, \zeta)}\right|_{x=1, \text { or } 2}$ represent the $P_{D}$ of both the search and the verification modes of the DDSS arrangements, respectively. The $H_{0}{ }^{\prime}(1)$ value of the DDSS scheme is expressed as:

$$
H_{0}{ }^{\prime}(1)=1+m \cdot P_{F 1}+K \cdot P_{F 1} \cdot P_{F 2},
$$

where $K$ denotes the false locking penalty factor expressed in terms of the number of chip intervals required by an auxiliary device for recognising that the code-tracking loop is still unlocked and $m$ represents the exponent of $z$ in the verification mode. Furthermore, $\left.P_{F x}\right|_{x=1, \text { or } 2}$ represent the $P_{F}$ in both the search and in the verification mode of the DDSS scheme, respectively.

\section{NUMERICAL SYSTEM PERFORMANCE RESULTS}

\section{TABLE I}

MAXIMUM SINR DEGRADATION INFLICTED BY BOTH THE DOPPLER SHIFT AND A $1000 \mathrm{HZ}$ FREQUENCY DRIFT IN CONJUNCTION WITH THE COHERENT INTEGRATION INTERVAL OF $N$ CHIP DURATIONS AT A CARRIER FREQUENCY OF $1.9 \mathrm{GHZ}$

\begin{tabular}{|l|l|l|l|l|l|}
\hline $\mathrm{N}($ Chips $)$ & 64 & 128 & 256 & 384 & 512 \\
\hline Degradation(dB) & 0.061 & 0.2449 & 0.9969 & 2.3144 & 4.3213 \\
\hline
\end{tabular}

In this section we will characterise the MAT performance of the cooperative MIMO aided DS-CDMA code acquisition scheme of Fig.2. In Table I we outlined the maximum SINR degradation imposed by both the Doppler shift and the frequency drift between the transmitter and receiver oscillators in conjunction with the coherent integration interval durations $\tau_{D}$ seen in the code acquisition schematic of Fig.2. 
The number of chips over which the integrator of Fig.2 sums the square of the envelope detector's output in both the search and the verification modes of DDSS is assumed to be 64 and 256 in the scenarios having $P=1$ and 2 RSs as well as $R=1,2$ and 4 receive antennas. The values of the link imbalance between the BS as well as the $P=1$ or $P=2 \mathrm{RS}(\mathrm{s})$ are assumed to be 0,3 and $6 \mathrm{~dB}$. Additionally, we assumed that there is no link imbalance between the two RSs. The spreading factor of the Walsh code to be acquired was selected to be 128 . The frequency drift was assumed to be $1000 \mathrm{~Hz}$ [9], whilst the carrier frequency was $1.9 \mathrm{GHz}$. As an example of a high mobile speed, it is reasonable to postulate $160 \mathrm{~km} / \mathrm{h}$. We also assumed that the sampling inaccuracy caused by having a finite, rather than infinitesimally low search step size of $\Delta=T_{c} / 2$ was $-0.91 \mathrm{~dB}$, which is a typical value for the search step size [9]. The total uncertainty region of code acquisition was assumed to entail $2 *\left(2^{15}-1\right)=65,534$ hypotheses. Finally, in the spirit of [10], the false locking penalty factor was assumed to be 1000 chip-durations. Both single-path and multi-path scenarios were considered. Each of the three paths of the multi-path scenario arrived with a relative time delay of one chip and they had a $3 \mathrm{~dB}$ lower magnitude for the first received path as well as $6 \mathrm{~dB}$ lower magnitude for the second and the third received paths than the LOS path of the single-path scenario, respectively. All paths were assumed to be present in a given search window. All the performance curves have been obtained at the optimum decision threshold of $E_{c} / I_{0}=-13 \mathrm{~dB}$ designed for the code acquisition scheme. The operational range of the two-RS scenario was assumed to be $3 \mathrm{~dB}$ lower than that of the single-RS one, because it is highly likely that the former may be situated near the cell-edge.

Fig.3 illustrates the achievable MAT versus SINR per chip performance of the DDSS code acquisition scheme parameterised with the grade of link imbalance and with the number of receive antennas for a single RS and a single path. In Figs. 3 and $5,{ }^{\prime} L 1^{\prime}$ denotes a singlepath scenario, whilst ' $L 3^{\prime}$ presents the scenario of encountering three paths in Figs. 4 and 6. The solid lines indicate the MAT curves recorded for RS-aided transmissions, whereas the dotted lines represent the MAT curves of our benchmarker. The link imbalance values of 0,3 , and $6 \mathrm{~dB}$ are clearly visible in all the figures. Observe in Fig. 3 that when the link imbalance is decreased, we experience an improved MAT performance. In a case of having no link imbalance, the MAT performance approaches that of having two receive antennas. On the other hand, in case of having $6 \mathrm{~dB}$ imbalance, only a marginal diversity gain is achieved, hence the attainable MAT performance improvement also becomes negligible. Fig. 4 characterises the MAT versus SINR per chip performance of the code acquisition scheme having exactly the same parameters as those considered in Fig.3, except for having three paths. Similarly to the conclusions of Fig.3, as the link imbalance is decreased, all the curves seen in Fig. 4 indicate an improved MAT peformance and vice versa. The above-mentioned conclusions explicitly demonstrate that employing a single RS is beneficial in terms of the achievable MAT performance, and as expected, the improvements depend on the value of the link imbalance, regardless whether single-path or multipath scenarios are considered. However, using a single RS cannot guarantee maintaining a high diversity gain due to the fluctuation of the RS's link quality. For comparison, Figs. 5 and 6 characterise the achievable MAT versus SINR per chip performance of the DDSS code acquisition scheme parameterised with both the value of link imbalance and the number of receive antennas, when considering two RSs in both a single-path (Fig.5) and in a three-path scenario (Fig.6), respectively. The results of Figs.5 and 6 also exhibit similar MAT performance trends, as shown in Figs.3 and 4, although the degree of achievable peformance improvements is higher than that of the single-RS scenario. To elaborate on the above results a little further, in case of having $0 \mathrm{~dB}$ imbalance the MAT performance attained becomes better than that of the benchmarker supported by $R=2$ or 4 receive antennas but no RS. Even a link imbalance of $6 \mathrm{~dB}$ is capable of ensuring a similar performance to that of the benchmarker. Therefore, owing to the random fluctuation of the link quality, the employment of two RSs might be beneficial in order to achieve a substantial performance improvement in RS-aided scenarios. In comparison to the detrimental effects of distributing the total transmit power over several co-located transmit antennas, as shown in [6], [7], based on Figs. 3 to 6 of this treatise, we infer that RS-aided scenarios benefit from higher multi-path diversity gains. In case of employing multiple co-located receive antennas at the MS, an additional receiver diversity gain is also achieved. Accordingly, by exploiting an appropriate combination of RS-aided transmissions and multiple co-located MS receive antennas, in the scenarios considered our scheme is capable of attaining a better performance than the benchmarker scheme benefitting from having four co-located MS receive antennas. It is worth mentioning furthermore that increasing the number of RSs used explicitly increases the number of independently fading multi-path components by a factor of $P$. However, this assumption implicitly expects that the extra transmit power is provided by the RS. The importance of post-initial acquisition [6] -which is capable of identifying the timing instants of the affordablecomplexity-dependent number of delayed and independently fading received signal paths to be combined by Rake receiver- is also worth exploring further. This is particularly so in the code acquisition schemes designed for ultra-wide band systems, owing to the inherent presence of an extremely high number of multi-path components [11]. Furthermore, due to the presence of an increased number of multipath components in the RS-aided scenario, either random search or non-consecutive search [11] combined with cell-by-cell detection may be applicable, which constitute the subject of our future research [11].

\section{CONCLUSION}

In this paper we considered the RS-aided distributed DL MIMOs and receive diversity of code acquisition schemes operating in the inter-cell synchronous CDMA DL. In contrast to the detrimental effects of sharing the total transmit power across multiple transmit antennas in co-located MIMO element based scenarios [6], [7], our analysis explicitly shows that in the absence of link imbalance, the achievable MAT performance of RS-aided distributed DL MIMOs approaches that of classic receive diversity, using multiple independently fading MS receive antenna elements, when considering the same total diversity order. On the other hand, in a case of having a high link imbalance, only marginal MAT performance gains may be achieved, regardless whether single-path or multi-path propagation scenarios are considered. Hence for the sake of exploiting the diversity benefits of RS-aided transmissions, the employment of at least two RSs might be recommended. When additionally invoking 
multiple co-located and yet independently fading receive antennas at the MS, further diversity gains may be achieved.

\section{REFERENCES}

[1] D. Gesbert, M. Shafi, D.S. Shiu, P.J. Smith, and A. Naguib, From Theory to Practice: An Overview of MIMO Space-Time Coded Wireless Systems, IEEE Journal on Selected Areas in Communications, vol. 21, NO.3, Issue 3, 2003, pp281-302.

[2] A. Sendonaris, E. Erkip and B. Aazhang, User Cooperation Diversity-Part I: System Description, IEEE Transactions on Communications, vol. 51, Issue 11, 2003, pp1927-1938.

[3] J. N. Laneman, D. N. C. Tse and G. W. Wornell, Cooperative Diversity in Wireless Networks: Efficient Protocols and Outage Behavior, IEEE Transactions on Information Theory, vol. 50, Issue 12, 2004, pp3062-3080.

[4] R. Pabst, B. H. Walke, D. C. Schultz, P. Herhold, H. Yanikomeroglu, S. Mukherjee, H. Viswanathan, M. Lott, W. Zirwas, M. Dohler, H. Aghvami, D. D. Falconer and G. P. Fettweis, Relay-based Deployment Concepts for Wireless and Mobile Broadband Radio, IEEE Communications Magazine, vol. 42, Issue 9, 2004, pp80-89.

[5] L. Hanzo, L-L Yang, E-L Kuan, K. Yen, Single- and MultiCarrier DS-CDMA, IV Multi-Carrier CDMA, Chapter 21, Initial Synchronization of DS-CDMA and MC-CDMA Systems, Wiley, 2003.

[6] S.H. Won and L. Hanzo, Initial and Post-Initial Acquisition in the Serial Search Based Noncoherent Multiple Transmit/Receive Antenna Aided DS-CDMA Downlink, Vehicular Technology Conference, vol. 5, 2006, pp2246-2250.

[7] S.H. Won and L. Hanzo, Differentially Coherent Code Acquisition in the MIMO-aided Multi-carrier DS-CDMA Downlink, IET Communications, vol. 1, Issue 4, 2007, pp662-670.

[8] L-L Yang, L. Hanzo, Serial Acquisition of DS-CDMA Signals in Multipath Fading Mobile Channels, IEEE Transactions on Vehicular Technology, vol. 50, Issue 2, 2001, pp617-628.

[9] A.J. Viterbi, CDMA: Principles of Spread Spectrum Communication, Chapter 3, Addison-Wesley, 1995.

[10] H.R. Park, Performance Analysis of a Double-Dwell Serial Search Technique for Cellular CDMA Networks in the Case of Multiple Pilot Signals, IEEE Transactions on Vehicular Technology, vol. 48, Issue 6, 1999, pp1819-1830.

[11] I. Ramachandran and S. Roy, On Acquisition of Wideband Directsequence Spread Spectrum Signals, IEEE Transactions on Wireless Communications, vol. 5, Issue 6, 2006, pp1537-1546.

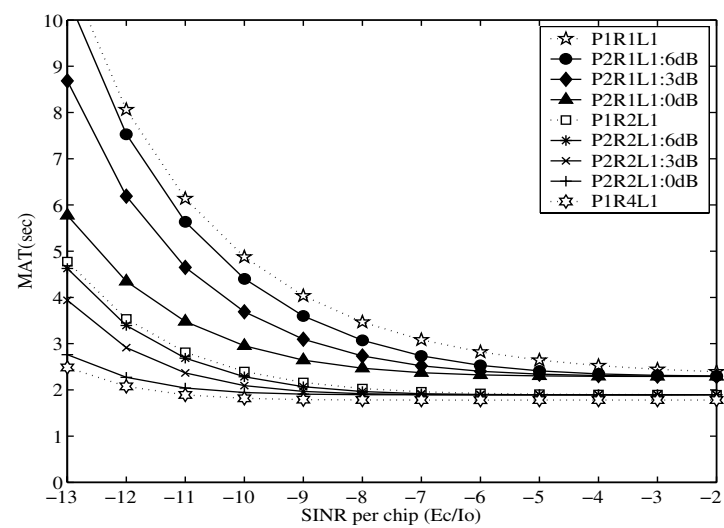

Fig. 3. MAT versus SINR per chip performance of the code acquisition system for DDSS parameterised with link imbalance and the number of receive antennas for one RS and a single path.

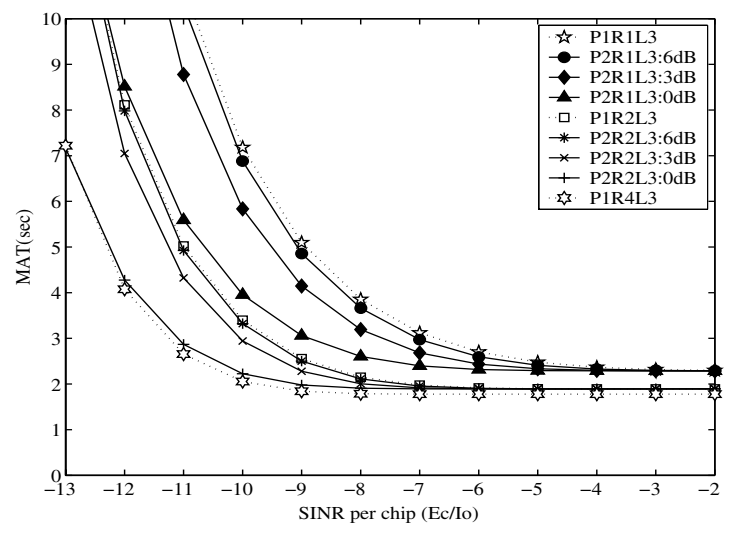

Fig. 4. MAT versus SINR per chip performance of the code acquisition system for DDSS parameterised with link imbalance and the number of receive antennas for one RS and three paths.

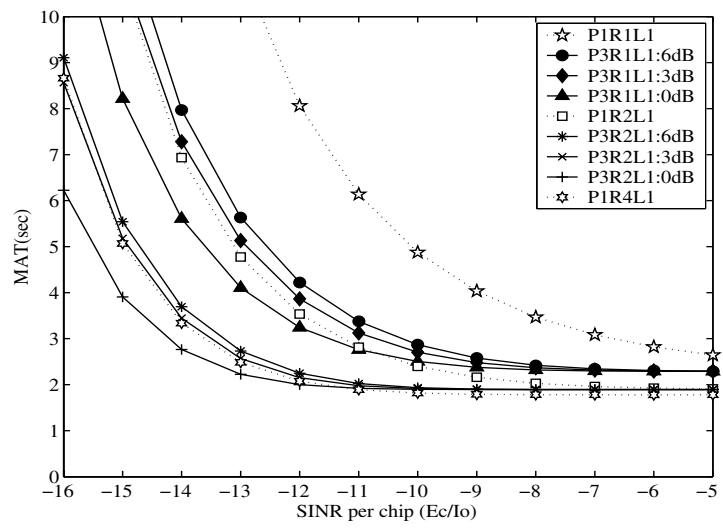

Fig. 5. MAT versus SINR per chip performance of the code acquisition system for DDSS parameterised with link imbalance and the number of receive antennas for two RSs and a single path.

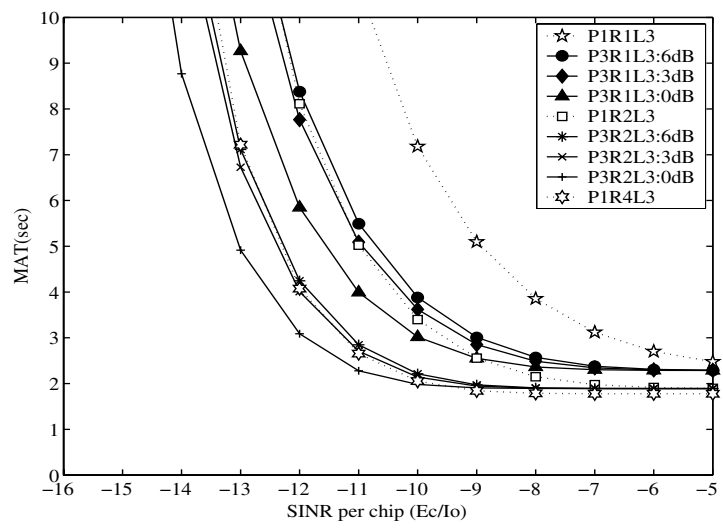

Fig. 6. MAT versus SINR per chip performance of the code acquisition system for DDSS parameterised with link imbalance and the number of receive antennas for two RSs and three paths. 